\title{
NOTES ON THE BREMEN CONGRESS ON ALCOHOLISM.
}

APRIL I4-I9, IgO3.

THE attendance at Bremen of $r, 400$ members at the Ninth International Anti-Alcoholic Congress afforded striking evidence of the widespread interest now taken in all that concerns the study and cure of inebriety. From almost every Government scientists or statesmen were accredited, and a considerable number of medical men took part in the proceedings.

A gathering of women who crowded the spacious Kunsthalle formed a fitting introduction to the more technical discussions on subsequent days. The German language was almost exclusively employed.

Beside the regular meetings of the Congress, there were evening meetings of a more social character, which proved in a convincing manner that even Germans may thoroughly enjoy themselves without alcohol. The Town Council of Bremen invited the delegates to a lunch in the fine old Ratshaus, above the famous Ratskeller, the Burgomaster presiding.

An exhibition of non-alcoholic wines, literature, etc., was also held during the Congress.

Only brief reference may be made to some of the more important communications :

Dr. Hueppe, of Prague, in discussing the "Influence of Alcohol on Physical Exercise," affirmed that moderate drinkers might perform muscular work more quickly than abstainers, but it was not done so thoroughly. The advantage of abstinence is demonstrated especially in prolonged and continuous muscular effort. The human system in some cases may become accustomed to the action of toxic agents and show no conspicuously bad results, but for the young, athletes, members of the army, and white men who live in the tropics, evidence has accumulated to indicate that abstinence is imperative. 
Dr. Legrain, of Paris, in speaking of the "Relationship of Alcoholism and Tuberculosis," contended that alcoholism rendered the individual incapable of adequate resistance to the attacks of the tubercle bacillus, and also, by lowering the moral sense, led to the neglect of the prophylactic influence of personal hygiene. The anti-tuberculosis campaign should be carried on by social and preventive measures. By curing a few consumptives tuberculosis cannot be eliminated, any more than drunkenness by the reclaiming of a few drunkards. Efforts to prevent the filthy habit of expectoration and measures to secure the isolation and efficient treatment of the phthisical will not alone conquer tuberculosis. Even sanatoria are of but limited benefit unless supported by general measures to prevent recurrence. Sound anti-alcoholic effort may accomplish much in diminishing the fearful prevalence of consumption.

In the discussion which followed several speakers objected to the idea that alcohol was the chief cause of tuberculosis, and one doctor contended that alcohol was beneficial in the last stages of consumption.

Drs. Ploetz and Rüdin, both of Berlin, in discussing the " Effect of Alcoholism in the Development of the Race," referred to the diminution in the birth-rate and rise in the death-rate, and sought to show that such was conspicuous wherever an increase occurred in the consumption of alcohol.

Dr. Lutoslawski, of Cracow, urged the importance of the moral and ethical aspect of total abstinence as distinguished from the mere hygienic.

Herr Smith, of Meindorff, showed that parental drunkenness frequently caused mental defects in the children, many of the firstborn offspring being feeble-minded in consequence, it was popularly believed, of their parents' indulgence during the wedding festivities.

Dr. Martins, of Rostock, in his paper on "The Misuse of Spirituous Drinks," held that the example of abstaining doctors was of great moral and hygienic value.

Dr. Forel, of Morges, in his paper on "Narcotics," pointed out that culture has made brainwork a far more important factor in earning a living than heretofore. The natural working of a healthy mind has no need of narcotics. In the discussion following this paper Count Skarzinski, of St. Petersburg, contended that prohibition laws do not always succeed, though they are strictly laid down both in Russia and in North America. The 
greatest good may be expected to result from the forces of education and self-reformation.

Dr. Fröhlich spoke of the great advance of total abstinence amongst the working-men of Vienna.

Drs. von Straus and Torney gave an account of the German union against the misuse of spirituous drinks.

Dr. Cramer, of Göttingen, in his paper on "Legal Measures for placing Drunkards under Care and Restraint," considered alcoholism a disease which must be scientifically treated. When restraint is enforced, it is often too late to secure full restoration. If no improvement is observed after six weeks of absolute abstinence, the case is almost hopeless. Restraint should be continued as long as possible, as after an apparent cure there is great danger of relapse.

Professor Endemann, of Halle, showed that by a law enacted in I9oI drunkards are to be placed in public or private asylums for the insane; but this applies to Prussia only. There is great need for inebriate homes throughout the provinces. A criminal drunkard should have the right to claim restraint in a sanatorium instead of other punishment.

Dr. Schmidt, of Bonn, held that nothing could be expected from the imprisonment of drunkards; they should be placed in suitable institutions, with constant medical superintendence, and such should be provided by the State.

Dr. Forel pointed out that formerly medical men had tried to cure drunkenness by alcoholic prescriptions. When in 1887 he started an asylum on Anglo-American lines he was much derided, but the results have justified the method.

Dr. Frank, of Münsterlingen, spoke on the "Medical Treatment of Inebriates," urging the necessity of treatment in accordance with physiological and psychological laws.

Dr. Fock, of Hamburg, contended that it is difficult to say when alcoholism begins. Abstaining families can do much good by taking in one or two inebriate patients. The physical inertia and mental languor of patients must be combated by providing them with work. Before being considered cured a patient should have visited public-houses without taking any intoxicant, and must be convinced of the paramount necessity of total abstinence for his very existence.

Dr. Keferstein, of Göttingen, in a paper on "Alcohol and Beer," quoted an old proverb, which says that "Beer is the $3-2$ 
strongest enemy of spirits." When Bauer and Bollinger were investigating "idiopathic" hypertrophy of the heart, they found it to be often due to overindulgence in beer. The theory that delirium tremens is not found amongst beer-drinkers has been proved to be false. In some districts more drunkards are made by beer than by spirit drinking.

Dr. Delbribck, of Bremen, showed that there is more alcohol than people imagine in beer. It is far from being the innocent food people formerly thought it. There is more drunkenness in France, Germany, and Bavaria, where beer and wine are consumed, than in Sweden and Norway, where alcohol is usually taken in the form of spirits.

Carefully prepared diagrams were displayed, which graphically illustrated the relative consumption of beer, wine, and spirits in various countries, and seemed to show that beer was often the pacemaker for spirits.

During the short time devoted to women's work in relation to the suppression of alcoholism, Miss Lammers, Miss Hoffmann, and three other ladies gave interesting addresses; and Mrs. Finlay received the thanks of many of the doctors present by suggesting the formation of a German League for Total-abstaining Nurses, like that federated to the English Women's Total Abstinence Union.

The Congress is to meet in 1905 at Buda-Pesth.

Grace A. Finlay.

Eleanor C. Finlay. 